\title{
A FUNCTIONAL DIFFERENTIAL EQUATION ARISING IN MODELLING OF CELL GROWTH
}

\author{
A. J. HALL ${ }^{1}$ AND G. C. WAKE ${ }^{2}$ \\ (Received 6 April 1988; revised 8 September 1988)
}

\begin{abstract}
A functional differential equation for the steady size distribution of a population is derived from the usual partial differential equation governing the size distribution, in the particular case where birth occurs by one individual of size $x$ dividing into $\alpha$ new individuals of size $x / \alpha$. This leads, in the case of constant growth and birth rate functions, to the functional differential equation $y^{\prime}(x)=-a y(x)+a \alpha y(\alpha x)$ together with the integral condition $\int_{0}^{\infty} y(x) d x=1$. We first look at a number of properties that any solution of this equation and boundary condition must have, and then proceed to find the unique solution by the method of Laplace transforms. Results from number theory on the infinite product found in the solution are presented, and it is shown that $y(x)$ tends to a normal distribution as $\alpha \rightarrow 1^{+}$.
\end{abstract}

\section{Introduction}

The following first-order functional differential equation arises when considering the growth and division of a spatially homogeneous population of cells (for example, see Sinko and Streifer [4]). Here $n(x, t)$ is the number density of cells of size $x$ at time $t, g(x, t)$ is the growth rate of these cells, and $b(x, t)$ is the rate at which cells of size $x$ are dividing, creating $\alpha$ new individuals of size $x / \alpha$. Of course, in this application $\alpha=2$, but consideration of other values of $\alpha$ is mathematically interesting ( $\alpha>1$ would correspond to a fission process and increasing population, $\alpha<1$ to a fusion process with decreasing population) without adding any significant complications. It is assumed that we are dealing with young, healthy

\footnotetext{
${ }^{1}$ Plant Physiology Division, DSIR, Palmerston North, New Zealand, and Department of Mathematics and Statistics, Massey University, Palmerston North, N.Z.

${ }^{2}$ Department of Mathematics and Statistics, Massey University, Palmerston North, N.Z.

(C) Copyright Australian Mathematical Society 1989, Serial-fee code 0334-2700/89
} 
tissue, and so mortality of cells is considered negligible. Accordingly, we have

$$
\frac{\partial}{\partial t}(n(x, t))+\frac{\partial}{\partial x}(g(x, t) n(x, t))=-b(x, t) n(x, t)+\alpha^{2} b(\alpha x, t) n(\alpha x, t) .
$$

It is clear from the comments above that for $\alpha \neq 1$ no steady-state solutions of this equation can exist, but there is considerable interest in the steady-size distribution (SSD) which can develop in a growing population if $g$ and $b$ are functions of $x$ alone. If we let $N(t)=\int_{0}^{\infty} n(x, t) d x$ be the total population at time $t$, then the size distribution of the population at time $t$ is $y(x, t)$, where $n(x, t)=y(x, t) N(t)$, and of course $\int_{0}^{\infty} y(x, t) d x=1$. If $y(x, t)$ is time-invariant (that is, dependent on $x$ only), then we write it as $y(x)$, where we must have $\int_{0}^{\infty} y(x) d x=1$, and call it an SSD. To find the appropriate differential equation for the SSD, we now apply the method of separation of variables, by substituting $n(x, t)=y(x) N(t)$ into (1), giving

$$
y(x) d N / d t+N(t) d(g(x) y(x)) / d x=-b(x) y(x) N(t)+\alpha^{2} b(\alpha x) y(\alpha x) N(t) .
$$

To find an expression for $d N / d t$ we can just integrate this equation over all $x$, giving

$$
\frac{d N}{d t}=N(t)(\alpha-1) \int_{0}^{\infty} b(x) y(x) d x
$$

where we have assumed that there is no flux of individuals at the boundaries, so $g(0) y(0)=g(\infty) y(\infty)=0$. This shows that $N(t)$ will vary exponentially if the population reaches its SSD. Substituting this expression into (2) and rearranging, we get this equation for the $\operatorname{SSD} y(x)$ :

$$
\frac{d(g(x) y(x))}{d x}=-\left[b(x)+(\alpha-1) \int_{0}^{\infty} b(x) y(x) d x\right] y(x)+\alpha^{2} b(\alpha x) y(\alpha x) .
$$

The case we consider here is the simplest non-trivial case, with $b(x)$ and $g(x)$ constants, say $b$ and $g$ respectively. While this case is not particularly realistic, its solution does offer some ideas which could be further explored in more realistic examples. The equation to solve then becomes, after simplification using $\int_{0}^{\infty} y(x) d x=1$,

$$
g d y / d x=-b \alpha y(x)+b \alpha^{2} y(\alpha x) .
$$

Finally, assuming $g \neq 0$ we can substitute $a=\alpha b / g$, forming the functional differential equation

$$
d y / d x=-a y(x)+a \alpha y(\alpha x)
$$

which we wish to solve subject to the condition

$$
\int_{0}^{\infty} y(x) d x=1
$$

Kato and McLeod [3] have studied the equation $y^{\prime}(x)=a y(\lambda x)+b y(x)$ subject to the initial condition $y(0)=y_{0}$. Here we show how with the aid of the integral 
condition the solution can be obtained directly by application of the Laplace transform technique, and that the application of the integral condition (7), instead of the more usual initial condition $y(0)=y_{0}$, leads to a solution which can be regarded as a probability density function (pdf), and has a number of interesting properties not previously investigated.

\section{Preliminaries}

We now proceed to study the solution of (6) and (7) in the region $x \geq 0$, ignoring the manner in which these equations were derived. As a first step in this process, we note that we may restrict our attention to $\alpha>0$, as $y(\alpha x)$ is undefined for $\alpha<0$, and $\alpha=0$ leads trivially to the simple solution $y=a e^{-a x}$. We may also assume $\alpha \neq 1$, as if $\alpha=1$ then (6) reduces immediately to $y^{\prime}(x)=0$ which has no solutions satisfying (7).

We shall first derive necessary properties of the solution, assuming at this stage that it exists.

$2.1 y(0)$ and $y(\infty)$

Condition (7) does not directly provide us with the initial condition $y(0)$, but we can show that the realistic assumption $y(0)=y(\infty)=0$ can be derived from (6) and (7) alone. If we integrate (6) and then take limits as $x \rightarrow \infty$ we get

$$
\begin{aligned}
\lim _{x \rightarrow \infty}(y(x)-y(0)) & =\lim _{x \rightarrow \infty}\left(-a \int_{0}^{x} y(s) d s+a \alpha \int_{0}^{x} y(\alpha s) d s\right), \\
\lim _{x \rightarrow \infty}(y(x)) & =-a+a+y(0),
\end{aligned}
$$

and so

$$
\lim _{x \rightarrow \infty}(y(x))=y(0)
$$

Now if $y(0) \neq 0$ then $y(\infty) \neq 0$ and so $\int_{0}^{\infty} y(x) d x$ does not converge, contradicting (7). Hence we must have

$$
y(0)=0
$$

and

$$
\lim _{x \rightarrow \infty}(y(x))=0 .
$$

The first of these results gives us the appropriate initial condition, and the second ensures that $y(x)$ is of exponential order so Laplace transforms can be applied.

It is interesting to note that if $y(0)=0$, all derivatives of $y$ are also zero at $x=0$. This can be shown iteratively by first substituting $x=0$ into (6) to show $y^{\prime}=0$, then differentiating (6) and substituting $x=0$ to show $y^{\prime \prime}=0$, and so on. Hence the solution is "infinitely flat" and therefore not analytic at $x=0$. 


\subsection{Restrictions on $y, \alpha$, and $a$}

Given $y(0)=y(\infty)=0$, and $\int_{0}^{\infty} y(x) d x=1>0$, it follows that $y(x)$ is positive for some range of $x$ values. It therefore has a maximum at some point $\bar{x}$ say, so from (6) we have $0=-a y(\bar{x})+a \alpha y(\alpha \bar{x})$, and so

$$
y(\alpha \bar{x})=y(\bar{x}) / \alpha
$$

Thus for $\alpha<1$ we would conclude $y(\alpha \bar{x})>y(\bar{x})$, contradicting the assumption that $y$ has its maximum at $\bar{x}$. Hence we must have

$$
\alpha>1 \text {, }
$$

and no SSD can exist in the fusion case, where $\alpha<1$.

We can show $y(x)>0(\forall x>0)$ by considering the variable $Z(x)=\int_{x}^{\infty} y(s) d s$. Integrating (6) leads to

$$
\begin{aligned}
\int_{x}^{\infty} y^{\prime}(s) d s & =-a Z(x)+a \alpha \int_{x}^{\infty} y(\alpha s) d s \\
Z^{\prime}(x) & =-a Z(x)+a Z(\alpha x) .
\end{aligned}
$$

This is subject of course to the conditions $Z(0)=1$ (from (7)) and $Z(\infty)=0$.

Assume for the moment that there exists a point $x_{1} \in(0, \infty)$ at which $Z^{\prime}\left(x_{1}\right)=-y\left(x_{1}\right)=0$, and $Z\left(x_{1}\right) \neq 0$. Then $Z\left(\alpha x_{1}\right)=Z\left(x_{1}\right)$ and there must be a point $x_{2} \geq \alpha x_{1}$ such that $Z^{\prime}\left(x_{2}\right)=0$ and $\left|Z\left(x_{2}\right)\right| \geq\left|Z\left(x_{1}\right)\right|$. Without loss of generality, assume $Z\left(x_{1}\right)>0$. Then one of the following must hold:

(a) $Z^{\prime}\left(\alpha x_{1}\right)>0$ in which case such an $x_{2}$ must exist, or

(b) $Z^{\prime}\left(\alpha x_{1}\right)=0$ so we can choose $x_{2}=\alpha x_{1}$, or

(c) $Z^{\prime}\left(\alpha x_{1}\right)<0$ so $\exists x^{*} \in\left(x_{1}, \alpha x_{1}\right)$ such that $Z^{\prime}\left(x^{*}\right)=0$ and $Z\left(x^{*}\right)>Z\left(x_{1}\right)$, so $Z\left(\alpha x^{*}\right)>Z\left(\alpha x_{1}\right)$ also. Then we have $\alpha x_{1}<\alpha x^{*}<\infty$ but $Z\left(\alpha x^{*}\right)>Z\left(\alpha x_{1}\right)$ and $Z\left(\alpha x^{*}\right)>Z(\infty)$. Thus there must be some point $x_{2}>\alpha x_{1}$ where $Z^{\prime}\left(x_{2}\right)>$ $Z\left(x_{1}\right)$ as required.

The argument for $Z\left(x_{1}\right)<0$ is almost identical. Hence we can find a sequence of points $\left(x_{n}\right) \rightarrow \infty$ as $n \rightarrow \infty$, at which $Z^{\prime}\left(x_{n}\right)=0$, whose images under the function $Z,\left(Z\left(x_{n}\right)\right) \nrightarrow 0$. This contradicts $Z(\infty)=0$, so it follows that $Z$ has no nonzero stationary points on $(0, \infty)$, so $Z$ is monotonically decreasing with $Z^{\prime}(x) \leq 0(\forall x>0)$. As $y(x)=-Z^{\prime}(x)$ we have for $x>0$,

$$
y(x) \geq 0,
$$

so $y(x)$ is a probability density function as desired.

Now (6) can be written as the integral equation

$$
y(x)=a \int_{x}^{\alpha x} y(s) d s
$$

so as $y(x)>0$, a necessary condition for the existence of an SSD must be

$$
a>0 \text {. }
$$




\subsection{Uniqueness of the solution}

Let there be two solutions to (6) and (7), $y_{1}(x)$ and $y_{2}(x)$. Then the variable $Z(x)=\int_{x}^{\infty}\left(y_{1}(s)-y_{2}(s)\right) d s$ must satisfy equation (13) but this time with the conditions $Z(0)=0$ and $Z(\infty)=0$. Now if $Z(x) \neq 0$ for any $x$, then there must be a stationary point $x_{1}$ of $Z$ such that $Z\left(x_{1}\right) \neq 0$, which by a similar argument to that given above leads to a contradiction. Hence we have $Z(x) \equiv 0$ so $Z^{\prime}(x) \equiv 0$ and

$$
y_{1}(x) \equiv y_{2}(x),
$$

and so there can be at most one solution to (6) and (7).

\subsection{Moments of the SSD}

Because of the integral condition (7), it is in fact easier to find the moments of the SSD than the function $y(x)$ itself. To find the $n$th moment of $y(x)$ about the origin, $\mu_{n}=\int_{0}^{\infty} x^{n} y(x) d x$, we multiply (6) by $x^{n}$ and integrate, so

so

$$
\int_{0}^{\infty} x^{n} y^{\prime}(x) d x=-a \mu_{n}+a \alpha \int_{0}^{\infty} x^{n} y(\alpha x) d x
$$

$$
-n \mu_{n-1}=-a \mu_{n}+a \mu_{n} / \alpha^{n}
$$

where we have used integration by parts on the left and the substitution $z=\alpha x$ on the right. This procedure is equivalent to taking the Mellin transform of each side if the equation. We now have an iterative equation for $\mu_{n}$ along with an initial value, given by (7),

$$
\mu_{n}=\frac{n}{a\left(1-\alpha^{-n}\right)} \mu_{n-1}, \quad \text { and } \quad \mu_{0}=1
$$

It now follows simply by induction that the $n$th moment of $y$ about the origin is given by

$$
\mu_{n}=n ! /\left(a^{n} \prod_{k=1}^{n}\left(1-\alpha^{-k}\right)\right) .
$$

Hence the mean cell size, $\mu$, is given by

$$
\mu=1 /\left(a\left(1-\alpha^{-1}\right)\right)
$$

and the variance of the cell size, $\dot{\sigma}^{2}$, is

$$
\sigma^{2}=1 /\left(a^{2}\left(1-\alpha^{-2}\right)\right)
$$

The third moment about the mean, $m_{3}$, can be shown from (18) to be

$$
m_{3}=2 /\left(a^{3}\left(1-\alpha^{-3}\right)\right)
$$

so the coefficient of skewness $\sqrt{\beta}_{1}$ is given by

$$
\sqrt{\beta}_{1}=m_{3} / \sigma^{3}=2(\alpha+1) \sqrt{\alpha^{2}-1} /\left(\alpha^{2}+\alpha+1\right) .
$$

Thus the coefficient of skewness is dependent on $\alpha$ only. 


\section{Solution of the SSD}

We now establish that equations (6) and (7) have a solution, which by Section 2.3 is unique. We first take Laplace transforms of each side of (6), remembering $y(0)=0$, giving

so

$$
p \bar{y}(p)=a \alpha \int_{0}^{\infty} e^{-p x} y(\alpha x) d x-a \bar{y}(p)
$$

$$
p \bar{y}(p)=a \bar{y}(p / \alpha)-a \bar{y}(p)
$$

Thus we have an iterative equation with an initial value given by the integral condition (7),

$$
\bar{y}(p)=\frac{1}{\left(1+\frac{p}{a}\right)} \bar{y}\left(\frac{p}{\alpha}\right),
$$

with

$$
\bar{y}(0)=1
$$

Iterating $n+1$ times, we get

$$
\bar{y}(p)=\frac{1}{\left(1+\frac{p}{a}\right)\left(1+\frac{p}{a \alpha}\right) \ldots\left(1+\frac{p}{a \alpha^{n}}\right)} \bar{y}\left(\frac{p}{\alpha^{n+1}}\right) .
$$

Now as $\alpha>1$ we know $\lim _{n \rightarrow \infty} \bar{y}\left(\frac{p}{\alpha^{n+1}}\right)=\bar{y}(0)=1$ so we get

$$
\bar{y}(p)=\lim _{N \rightarrow \infty} \prod_{n=0}^{N} \frac{1}{\left(1+\alpha^{-n p} \frac{a}{a}\right)} .
$$

Expressing this product as a sum of partial fractions we can write

$$
\prod_{n=0}^{N} \frac{1}{\left(1+\alpha^{-n p}\right)}=\sum_{n=0}^{N} \frac{Q_{n}\left(-a \alpha^{n}\right)}{\left(1+\alpha^{-n} \underset{a}{p}\right)}
$$

where $Q_{n}(p)$ is the product on the left hand side of this equation excluding the factor involving $\alpha^{-n}$. Thus,

$$
Q_{n}(p)=\frac{1}{\left(1+\frac{p}{a}\right)\left(1+\alpha^{-1} \frac{p}{a}\right) \ldots\left(1+\alpha^{-(n-1)} \frac{p}{a}\right)\left(1+\alpha^{-(n+1)} \frac{p}{a}\right) \ldots\left(1+\alpha^{\left.-N \frac{p}{a}\right)}\right.}
$$

and

$$
Q_{n}\left(-a \alpha^{n}\right)=\frac{1}{\left(1-\alpha^{n}\right) \ldots(1-\alpha)\left(1-\alpha^{-1}\right)\left(1-\alpha^{-2}\right) \ldots\left(1-\alpha^{-(N-n)}\right)}
$$

Simplifying and taking limits, we get

$$
\bar{y}(p)=\frac{1}{K} \sum_{n=0}^{\infty} \frac{1}{(1-\alpha)\left(1-\alpha^{2}\right) \ldots\left(1-\alpha^{n}\right)} \frac{1}{\left(1+\alpha^{-n} p / a\right)}
$$


where the product in the denominator is given the value 1 when $n=0$, and

$$
K=\prod_{n=1}^{\infty}\left(1-\alpha^{-n}\right)
$$

Now taking the inverse Laplace transform term by term we get

$$
y(x)=\frac{1}{K} \sum_{n=0}^{\infty} \frac{1}{(1-\alpha)\left(1-\alpha^{2}\right) \ldots\left(1-\alpha^{n}\right)} a \alpha^{n} e^{-a \alpha^{n} x} .
$$

Now remembering $\alpha>1$,

$$
y(x)=\frac{a}{K} \sum_{n=0}^{\infty} \frac{(-1)^{n} \alpha^{n} e^{-a \alpha^{n} x}}{(\alpha-1)\left(\alpha^{2}-1\right) \ldots\left(\alpha^{n}-1\right)},
$$

which is the solution given by Kato and McLeod [3] in a slightly different form.

\section{Discussion}

\subsection{The constant $K$}

The infinite product in (32), which gives the value of $K$, has been studied in some depth by number theorists and others. Perhaps the best known result involving this product, and one which offers a rapidly convergent way of calculating $K$, is Euler's Pentagonal Number Theorem (Andrews [2]). This is

$$
\begin{aligned}
\prod_{n=1}^{\infty}\left(1-q^{n}\right) & =\sum_{n=-\infty}^{\infty}(-1)^{n} q^{n(3 n-1) / 2} \\
& =1-\left(q+q^{2}\right)+\left(q^{5}+q^{7}\right)-\left(q^{12}+q^{15}\right)+\ldots
\end{aligned}
$$

where $|q|<1$, so we have

$$
K=1-\left(\alpha^{-1}+\alpha^{-2}\right)+\left(\alpha^{-5}+\alpha^{-7}\right)-\left(\alpha^{-12}+\alpha^{-15}\right)+\ldots
$$

Another interesting (and rapidly convergent) series for $K$ can be deduced by substituting $p=0$ in (31) and using (25), giving

$$
K=\sum_{n=0}^{\infty} \frac{(-1)^{n}}{(\alpha-1)\left(\alpha^{2}-1\right) \ldots\left(\alpha^{n}-1\right)}
$$

which could also have been deduced by substitution of $q=\alpha^{-1}$ and $z=-q$ in

$$
\sum_{n=0}^{\infty} \frac{q^{n(n-1) / 2} z^{n}}{(1-q)\left(1-q^{2}\right) \ldots\left(1-q^{n}\right)}=\prod_{n=0}^{\infty}\left(1+z q^{n}\right)
$$

which is also given by Andrews [2]. Once again, we are using the convention that the product in the denominator is given the value 1 when $n=0$. 
Another expression for $K$, but one we do not actually use here, can be derived from a formula involving Jacobian elliptic functions. Formula 16.37.1 in Abramowitz and Stegun [1] leads to

$$
\prod_{n=1}^{\infty}\left(1-q^{2 n}\right)=\pi\left[\frac{m(m-1)}{16 q}\right]^{\frac{1}{12}},
$$

which could be used to calculate $K$ if we make the substitution $q=\alpha^{-\frac{1}{2}}$. This transfers the problem to relating the elliptic function parameter $m$ to the nome $q$, which is possible via Landen's transformation.

\subsection{Properties of the solution}

From a cursory inspection of (34) alone, it is not at all immediately obvious that $y(x)$ satisfies all the properties that we deduced earlier it must have. For example, substituting $x=0$ in equation (34) leads to

$$
y(0)=\frac{a}{K} \sum_{n=0}^{\infty} \frac{(-1)^{n} \alpha^{n}}{(\alpha-1)\left(\alpha^{2}-1\right) \ldots\left(\alpha^{n}-1\right)} .
$$

To show this is equal to zero, we need to write the $\alpha^{n}$ in the numerator as $\left(\alpha^{n}-1\right)+1$ and combine adjacent terms leading to

$$
\sum_{n=0}^{\infty} \frac{(-1)^{n} \alpha^{n}}{(\alpha-1)\left(\alpha^{2}-1\right) \ldots\left(\alpha^{n}-1\right)}=\lim _{N \rightarrow \infty}\left[\frac{(-1)^{N}}{(\alpha-1)\left(\alpha^{2}-1\right) \ldots\left(\alpha^{N}-1\right)}\right] .
$$

As $\alpha>1$, the denominator grows without limit as $N \rightarrow \infty$ and we have

$$
y(0)=0 \text {. }
$$

To verify (7) by substitution, we need to first interchange the integral and summation signs (which is valid in view of the uniform convergence of the series), and then apply (37), so that

$$
\begin{aligned}
\int_{0}^{\infty} y(x) d x & =\frac{a}{K} \int_{0}^{\infty} \sum_{n=0}^{\infty} \frac{(-1)^{n} \alpha^{n} e^{-a \alpha^{n} x}}{(\alpha-1)\left(\alpha^{2}-1\right) \ldots\left(\alpha^{n}-1\right)} d x \\
& =\frac{1}{K} \sum_{n=0}^{\infty} \frac{(-1)^{n}}{(\alpha-1)\left(\alpha^{2}-1\right) \ldots\left(\alpha^{n}-1\right)}
\end{aligned}
$$

so

$$
\int_{0}^{\infty} y(x) d x=1
$$

as required. Verifying (18) by direct substitution in $\mu_{n}=\int_{0}^{\infty} x^{n} y(x) d x$ requires a similar interchange of order, then application of (38) with $z=-\alpha^{-(n+1)}$ and $q=\alpha^{-1}$, and then finally substitution for $K$ from (32).

\subsection{An example}

Consider a population of cells undergoing binary fission $(\alpha=2)$ with a constant 


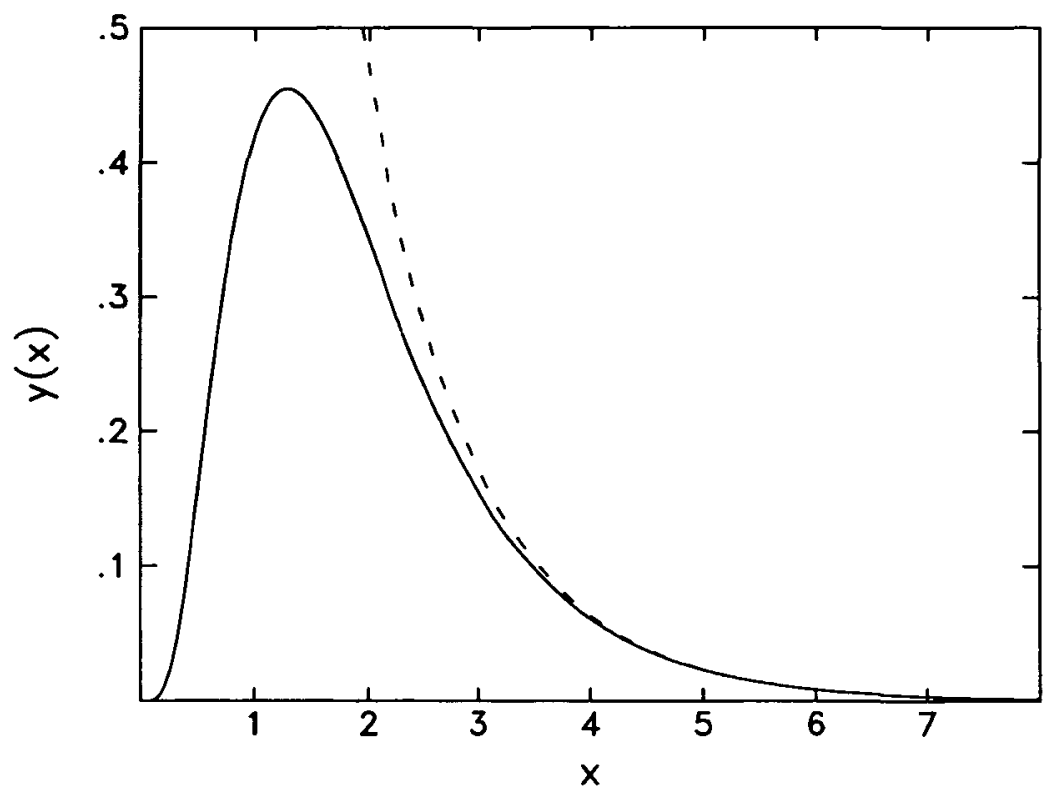

Figure 1. The solution for $a=1$ and $\alpha=2$. The dashed line is the graph of $y(x)=a e^{-a x} / K$.

growth rate $g$ of 0.2 size units per second, and a division rate $b$ of 0.1 per cell per second. Then $a=b \alpha / g=1$ and $K=\prod_{n=0}^{\infty}\left(1-\frac{1}{2}^{n}\right)=1-\left(\frac{1}{2}+\frac{1}{4}\right)+\left(\frac{1}{32}+\frac{1}{128}\right)-$ $\left(\frac{1}{4096}+\frac{1}{32768}\right)+\cdots \approx .288788$. The mean, variance, and coefficient of skewness of the SSD are obtained by substitution in (19), (20), and (22) respectively, giving $\mu=2, \sigma^{2}=4 / 3 \approx 1.3333$, and $\sqrt{\beta}_{1}=6 \sqrt{3} / 7 \approx 1.4846$.

Figure 1 shows the shape of the SSD function $y(x)$ in this case as given by (34).

The qualitative features of Figure 1 remain for all $a$ and $\alpha$. The parameter $a$ acts as a simple scaling factor for $x$ (the substitutions $t=a x$ and $z(t)=y(x)$ make (6) independent of $a$ ). Equation (22) shows that the coefficient of skewness is always positive, increasing from 0 to 2 as $\alpha$ increases from 1 to $\infty$.

\subsection{The solution for extreme values of $\alpha$}

In the light of the comments at the end of the example, it is worth considering the form of the solution when $\alpha \rightarrow \infty$ and $\alpha \rightarrow 1^{+}$. The first is easy-if we write (34) in the form given in Kato and McLeod [3], namely

$$
y(x)=\frac{a}{K} e^{-a x}\left(1+\sum_{n=1}^{\infty} \frac{(-1)^{n} \alpha^{n} e^{-a\left(\alpha^{n}-1\right) x}}{(\alpha-1)\left(\alpha^{2}-1\right) \ldots\left(\alpha^{n}-1\right)}\right),
$$

then it is clear that for all $\alpha, y(x) \approx a e^{-a x} / K$ for large $x$, and that in particular, for all $x>0$ we have

$$
\lim _{\alpha \rightarrow \infty} y(x)=a e^{-a x} / K
$$


Hence in the limit as $\alpha \rightarrow \infty, y(x)$ approaches an exponential pdf with parameter a.

The limit as $\alpha \rightarrow 1^{+}$is much more difficult. Inspection of (34) shows that each term in the series becomes infinite as $\alpha \rightarrow 1^{+}$, and (18) shows that all the moments become infinite as well. This suggests that we "normalise" the equation using the transformation

$$
z=(x-\mu) / \sigma
$$

and

$$
Y(z)=\sigma y(x)
$$

where $\mu$ and $\sigma$ are given by (19) and (20).

Figure 2, which shows $Y(z)$ plotted for various values of $\alpha$, suggests that in the limit as $\alpha \rightarrow 1^{+}, Y(z)$ approaches the pdf of the standard normal distribution $N(0,1)$. We now have

$$
\int_{-\mu / \sigma}^{\infty} Y(z) d z \approx 1, \quad y^{\prime}(x)=Y^{\prime}(z) / \sigma^{2}
$$

and

$$
y(\alpha x)=Y(\alpha z+(\alpha-1) \mu / \sigma) / \sigma,
$$

so substituting into (6) we obtain $Y^{\prime}(z) / \sigma^{2}=-a Y(z) / \sigma+a \alpha Y\left(\alpha z+(\alpha-1) \frac{\mu}{\sigma}\right) / \sigma$.

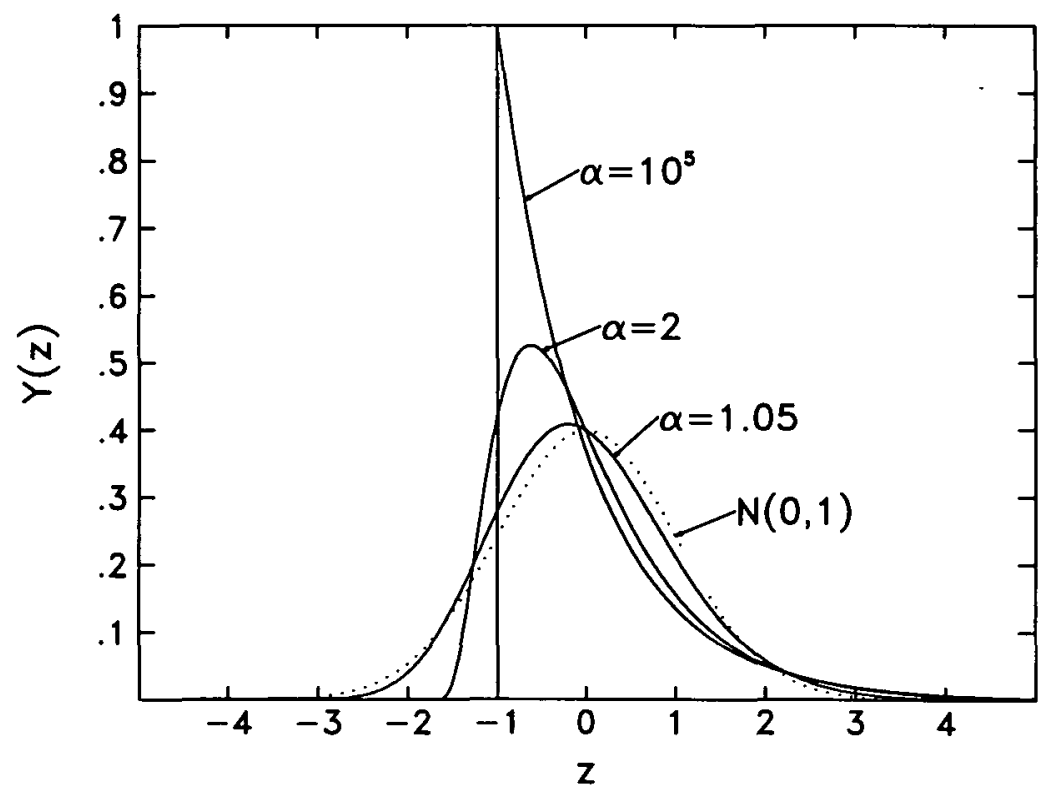

FIGURE 2. The normalised pdf $Y(z)=\sigma y(\sigma z+\mu)$ for various values of $\alpha$. The dashed line is the pdf of the standard normal distribution. 
Now substituting using (19) and (20) we get the "normalised" equation

$$
Y^{\prime}(z)=\left(\alpha / \sqrt{\alpha^{2}-1}\right)\left[\alpha Y\left(\alpha z+\sqrt{\alpha^{2}-1}\right)-Y(z)\right] .
$$

Now to find out what happens as $\alpha \rightarrow 1^{+}$, we expand the $Y\left(\alpha z+\sqrt{\alpha^{2}-1}\right)$ as a Taylor series about $Y(z)$ up to all terms of order $(\alpha-1)$, giving

$$
Y\left(\alpha z+\sqrt{\alpha^{2}-1}\right)=Y\left(z+\left[(\alpha-1) z+\sqrt{\alpha^{2}-1}\right]\right),
$$

so

$$
Y\left(\alpha z+\sqrt{\alpha^{2}-1}\right) \approx Y(z)+\left[(\alpha-1) z+\sqrt{\alpha^{2}-1}\right] Y^{\prime}(z)+\left(\alpha^{2}-1\right) / 2 Y^{\prime \prime}(z) .
$$

Substituting this expression into (48) and simplifying, we get

$$
\alpha Y(z)+\alpha^{2} z Y^{\prime}(z)+\left[(\alpha+1) \sqrt{\alpha^{2}-1}\right] Y^{\prime}(z)+\left(\alpha^{2}(\alpha+1) / 2\right) Y^{\prime \prime}(z)=0,
$$

which on substitution of $\alpha=1$ becomes the second order differential equation

$$
Y^{\prime \prime}(z)+z Y^{\prime}(z)+Y(z)=0,
$$

subject to the integral condition

$$
\int_{-\infty}^{+\infty} Y(z) d z=1
$$

It is trivial to show that

$$
Y(z)=Y_{1}(z)=e^{-z^{2} / 2}
$$

is a solution of (50). A second (independent) solution can then be found by substituting say $Y(z)=Y_{2}(z)=U(z) Y_{1}(z)$, leading (after cancellation of $Y_{1}(x)$ ) to

$$
U^{\prime \prime}(z)-z U^{\prime}(z)=0 .
$$

Hence we may choose

$$
U^{\prime}(z)=e^{z^{2} / 2}, \quad \text { and } \quad Y_{2}(z)=e^{-z^{2} / 2} \int_{0}^{z} e^{s^{2} / 2} d s .
$$

The general solution of $(50)$ is then

$$
Y(z)=C_{1} Y_{1}(z)+C_{2} Y_{2}(z)
$$

where $C_{1}$ and $C_{2}$ are arbitrary constants, and $Y_{1}(z)$ and $Y_{2}(z)$ are given by (52) and (54) respectively. Now applying (51), we note that $\int_{c}^{\infty} Y_{2}(z) d z$ is infinite for all $c$, so we must have $C_{2}=0$. Hence $C_{1}=1 / \sqrt{2 \pi}$, and the only solution to (50) and (51) is

$$
Y(z)=e^{-z^{2} / 2} / \sqrt{2 \pi}
$$

which is the pdf of the standard normal distribution as expected. 
Consideration of the neglected terms in $(\alpha-1)$ shows that we can write, for all $\alpha$ close to 1,

$$
Y(z)=e^{-z^{2} / 2}(1+\mathrm{O}(\sqrt{\alpha-1})) / \sqrt{2 \pi} .
$$

Reverting to the original coordinates, we can then say that for $\alpha$ close to 1 ,

$$
y(x)=e^{-(x-\mu)^{2} / 2 \sigma^{2}}(1+\mathrm{O}(\sqrt{\alpha-1})) / \sqrt{2 \pi \sigma^{2}}
$$

where $\mu$ and $\sigma^{2}$ are given by (19) and (20) respectively. Thus as $\alpha \rightarrow 1^{+}, y(x)$ approaches the pdf of a normal distribution.

4.5 The form of the time-dependent number density $n(x, t)$

Substituting $b(x)=b$ in (3) gives $d N / d t=N(t)(\alpha-1) b$, and so

$$
N(t)=N_{0} e^{(\alpha-1) b t}
$$

where $N_{0}=N(0)$. We can now substitute (34) and (58) into $n(x, t)=N(t) y(x)$ to obtain the separable form of $n(x, t)$ which applies when the size distribution is given by the SSD

$$
n(x, t)=N(t) y(x)=\frac{a N_{0}}{K} e^{(\alpha-1) b t} \sum_{n=0}^{\infty} \frac{(-1)^{n} \alpha^{n} e^{-a \alpha^{n} x}}{(\alpha-1)\left(\alpha^{2}-1\right) \ldots\left(\alpha^{n}-1\right)} .
$$

\section{Acknowledgement}

Thanks are due to J. F. Harper (Victoria University of Wellington) for suggesting the transformation used in (13), and to a referee for a number of constructive suggestions, including the use of the Mellin transformation in Section 2.4 and the connection with Jacobian elliptic functions in Section 4.1.

\section{References}

[1] M. Abramowitz and I. A. Stegun, Handbook of mathematical functions (Dover, New York, 1965).

[2] G. E. Andrews, Number theory (W. B. Saunders, Phil., 1971).

[3] T. Kato and J. B. McLeod, "The functional differential equation $y^{\prime}(x)=a y(\lambda x)+b y(x)$ ", Bull. Amer. Math. Soc. 77 (1971) 891-937.

[4] J. W. Sinko and W. Streifer, "A model for populations reproducing by fission", Ecology 52 (1971) 330-335. 